\title{
Evaluation of vestibulocochlear nerves in cases with acoustic schwannoma by direct sagittal oblique 3D-FIESTA MR imaging
}

\author{
Hidenobu Taiji*, Yasuhide Okamoto and Jin Kanzaki \\ Department of Otorhinolaryngology, Saiseikai Central Hospital, 1-4-17, Mita, Minato-ku, Tokyo, Japan
}

\begin{abstract}
Oblique sagittal MRI with 3D-FIESTA (3-D fast imaging employing steady-state acquisition) sequences can be obtained through the internal auditory canal (IAC) without relying on multi-planar reconstructions. The high spatial resolution direct sagittal oblique imaging technique is useful for the identification of the four intracanalicular nerves. This work included one case of IAC tumor and two cases of cerebellopontine angle (CPA) tumors. MR imaging using a 3D-FIESTA sequence was performed in the oblique sagittal plane perpendicular to the course of the IAC. The direct sagittal oblique imaging technique allowed clear delineation of the individual nerves of the IAC. In a case of acoustic schwannoma, which was surgically proven to originate from the inferior vestibular nerve (IVN), the results of vestibular assessment indicated an IVN pathology. Acquired cochlear nerve hypoplasia with moderate hearing loss was found in a case of CPA tumor.
\end{abstract}

\section{Introduction}

Technological advances in MRI have led to the development of a new main diagnostic method for acoustic schwannoma. Thin section contrast-enhanced T1-weighted (T1WI) MRI is currently the accepted standard imaging approach, although recently the T2-weighted (T2WI) 3D-FIESTA (three-dimensional fast imaging employing steadystate acquisition) sequence has been introduced. The 3D-FIESTA sequence achieves the accuracy of T1-weighted sequences acquired with gadolinium, without the need for exogenous contrast agents [1]. Current steady-state free precession (SSFP) sequences, such as FIESTA and constructive interference steady state (CISS), allow high spatial resolution and a clear depiction of the facial and vestibulocochlear nerves, facilitating accurate detection of small tumors in the cerebellopontine angle (CPA) and internal auditory canal (IAC) [1]. The acquisition of an oblique sagittal SSFP sequence perpendicular to the course of the IAC provides a high-resolution image of the IAC, and has been reported as being appropriate for the diagnosis of cochlear nerve $(\mathrm{CN})$ hypoplasia [2]. The present work reports on the technique of direct oblique sagittal 3D-FIESTA MR imaging through the IAC, including examining the MRI findings of intra-canalicular nerves in acoustic schwannoma, and comparing the findings with those of audiological and vestibular examinations.

\section{Materials and methods}

\section{Patient population}

Between July 2013 and May 2016, 43 patients (29 male, 14 female; mean age at testing, 56.4 years; age range, 29 to 85 years) who presented with sudden or progressive unilateral sensorineural hearing loss (SNHL) at our hospital were examined using high-resolution MRI, including 3D-FIESTA sequences. Pathological findings on MRI were found in four cases (one IAC tumor; two cerebellopontine angle (CPA) tumors; one cerebellar infarction in the anterior inferior cerebellar artery territory). Of these, the patient with an IAC tumor and the two patients with CPA tumors were included in this study, and the results of audiological and vestibular examinations were compared with MRI findings. The IAC tumor (Patient 1) was surgically resected because of rapid growth due to cystic degeneration and was pathologically diagnosed as acoustic schwannoma. From the surgical findings, it was identified as originating from the inferior vestibular nerve (IVN). In Patients 2 and 3, a provisional diagnosis of the CPA tumor was acoustic schwannoma, and no tumor growth was observed were over 2 years.

\section{Imaging}

MR images were obtained using a 1.5 Tesla system (Signa HDX, GE, Milwaukee, WI, USA), and included a T2WI 3D-FIESTA sequence obtained in oblique sagittal slices through the IAC. The 3D-FIESTA sequence was performed using the following parameters: repetition time $(\mathrm{TR})=7.6 \mathrm{~s}$, echo time $(\mathrm{TE})=2.9 \mathrm{~ms}$, flip angle $=65^{\circ}$, slice thickness $=0.5$ $\mathrm{mm}$, field of view $=10 \mathrm{~cm} /$ image, matrix $=288 \times 256$, in-plane resolution $100 \times 100 \mathrm{~mm}$, and number of excitations $(\mathrm{NEX})=3$. The 3D-FIESTA images were obtained in the oblique sagittal plane, perpendicular to the course of the IAC, without using multi-planar reconstruction (MPR). An oblique sagittal image of a normal IAC is demonstrated in Figure 1B (Patient 1, unaffected side). All four intra-canalicular nerves (facial nerve $[\mathrm{FN}], \mathrm{CN}$, superior vestibular nerve [SVN], and IVN) were clearly identifiable in all 43 subjects except the tumor-involved side of the three patients.

Correspondence to: Hidenobu Taiji, MD, Department of Otorhinolaryngology, Saiseikai Central Hospital, 1-4-17, Mita, Minato-ku, Tokyo 108-0073, Japan, Tel: +81 33451 8211; Fax: +81 33451 6102; E-mail: taijih5@gmail.com

Key words: MR imaging, 3D-FIESTA, acoustic schwannoma, speech audiometry, cochlear nerve hypoplasia

Received: June 03, 2017; Accepted: June 20, 2017; Published: June 24, 2017 


\section{Audiological and vestibular assessment}

Pure tone audiometry was evaluated using a three-tone-average, formulated as $(a+b+c) / 3$, where $a, b$, and $c$ were hearing levels at 0.5 , 1 , and $2 \mathrm{kHz}$, respectively. Furthermore, testing was also performed for distortion product otoacoustic emissions (DPOAEs), auditory brainstem responses (ABRs), speech audiometry, caloric reflex, and ocular vestibular-evoked myogenic potentials (oVEMPs).

DPOAEs were measured for pairs of primary tones ( $f 1$ and $f 2$ ) in all subjects, with a fixed ratio of $\mathrm{f} 2 / \mathrm{f} 1=1.2$, and fixed sound pressure levels (SPL) of $65 \mathrm{~dB}$ (L1) and $55 \mathrm{~dB}$ (L2), obtained using an ILO292USB system (Otodynamics, Hatfield, United Kingdom). The presence of DPOAE was defined as a noise floor (signal to noise ratio; SNR) of $\geq 3 \mathrm{~dB}$.

ABRs and oVEMPs were recorded using a Neuropack M1 system (MEB-9204; Nihon Kohden, Tokyo, Japan). ABRs were performed by presenting $0.1 \mathrm{~ms}$ clicks with alternating polarity at a repetition rate of $10 \mathrm{~Hz}$ and a maximum intensity of $105 \mathrm{~dB} \mathrm{nHL}$ (decibel above normal adult hearing level). The wave $\mathrm{V}$ detection thresholds and the interaural differences in the latencies of wave V (IT5) at $80 \mathrm{~dB} n H L$ were examined. The normative value of IT5 at $80 \mathrm{~dB} \mathrm{nHL}$ is $<0.4 \mathrm{~ms}$. The muscle below the eye was selected as the target for recording the oVEMPs stimulated by a $500 \mathrm{~Hz}$ tone burst $(100 \mathrm{~dB} \mathrm{nHL}, 1 \mathrm{~ms}$ rise/fall time, $10 \mathrm{~Hz}$ repetition rate). The presence of an oVEMP was defined by the appearance of the first negative component at a latency of about $10 \mathrm{~ms}$ (n10).

Speech audiometry was performed in all subjects. The maximum word recognition scores were evaluated according to the percentage of correct answers out of twenty words, using the Japanese word list 67$\mathrm{S}$. In normal hearing subjects, the maximum word recognition scores using this word list are usually $\geq 90 \%$.

Alternate binaural bithermal caloric testing was performed using cool water $\left(30^{\circ} \mathrm{C}\right)$ and warm water $\left(40^{\circ} \mathrm{C}\right)$. Eye movements were recorded using an ENG system (NY-50, Rion, Tokyo, Japan). Directional preponderance (DP) was determined for the peak velocity of each irrigation using the following formula:

$\mathrm{DP}=\left[\left(\mathrm{R} 30^{\circ}+\mathrm{L} 44^{\circ}\right)-\left(\mathrm{R} 44^{\circ}+\mathrm{L} 30^{\circ}\right)\right] /\left(\mathrm{R} 30^{\circ}+\mathrm{L} 44^{\circ}+\mathrm{R} 44^{\circ}+\mathrm{L} 30^{\circ}\right) \times 100(\%)$.

A DP value of greater than $25 \%$ was considered to be pathological $(\mathrm{DP}+)$. The absence of a caloric nystagmus during the irrigation of ice water $\left(0^{\circ} \mathrm{C}\right)$ was defined as canal paralysis.

\section{Report of cases}

The identification rates for the intra-canalicular nerves (FN, CN, SVN, and IVN) on oblique sagittal 3D-FIESTA MR images of the IAC were $100 \%$ in the unaffected side in all 43 patients. A summary of the findings for the three patients with acoustic schwannoma are presented in Table 1. Figures 1 and 2 show the MRI findings and the results of the audiological examinations in Patient 1 (IAC tumor), while Figures 3 and 4 show them for Patient 2 (CPA tumor), and Figures 5 and 6 for Patient 3 (CPA tumor).

The axial T2WI MR image of Patient 1 shows a filling defect within the high-signal cerebral spinal fluid in the right IAC (Figure 1A). The 3D-FIESTA oblique sagittal MR image at the level of the right porus acusticus clearly reveals a $5 \mathrm{~mm}$ intra-canalicular tumor (Figure $1 \mathrm{C}$ ). The 3D-FIESTA oblique sagittal MR image at the lateral IAC (findus) clearly displays four nerves in the left side (Figure 1B), while the nerves in the right side are not clearly delineable, and the IVN is not visible
(Figure 1D). The long diameter [3] of the right $\mathrm{CN}$ was $0.8 \mathrm{~mm}$. The axial T2WI MR images of Patients 2 and 3 show a $15 \mathrm{~mm}$ CPA mass and an enlarged canal on the left side (Figure 3A- Figure 5A). In Patient 2, the $\mathrm{CN}$ and IVN are undetectable on the 3D-FIESTA oblique sagittal MR image through the left IAC (Figure 3B). The 3D-FIESTA oblique sagittal MR image of Patient 3 shows atrophic intra-canalicular nerves (Figure 5B), with the long diameter of the right $\mathrm{CN}$ being $0.6 \mathrm{~mm}$.

The hearing levels of the affected ears in the three cases ranged from 35 to $62 \mathrm{~dB}$ (Table 1). The audiogram shapes (Figure 2A, 4A, 6A) were low-frequency sloping (Patient 1 ) and high-frequency sloping (Patients 2 and 3). The maximum word recognition scores of the affected ears ranged from $45 \%$ to $70 \%$ (Table 1). DPOAEs were present at $2-7 \mathrm{kHz}$ in one ear (Patient 1), but were undetectable in the affected ears of Patients 2 and 3. ABR testing was performed in Patients 1 and 2 (Table 1). In comparison with normal values for $1000-4000 \mathrm{~Hz}$ pure tone hearing levels, the wave $\mathrm{V}$ threshold of the affected ear of Patient 1 was elevated ( $60 \mathrm{~dB} \mathrm{nHL})$. The wave $V$ latencies in the affected ears of Patients 1 and 2 were prolonged (the values of IT5 were 1.9 and $1.2 \mathrm{~ms}$, respectively). Caloric testing was performed in Patients 1 and 2, and abnormal caloric results were obtained in both cases (Table 1). The DP value in Patient 1 was 39\%, which represents significant asymmetry and a suggestion of canal paresis in the affected side. A caloric response was absent (canal paralysis) in the affected ear of Patient 2. The oVEMPs were tested at $100 \mathrm{~dB}$ nHL in Patients 1 and 2, and were present in both sides of both cases (Table 1).

\section{Discussion}

T2WI MR imaging sequences are highly sensitive for the screening for CPA/IAC lesions, without the use of exogenous contrast agents.
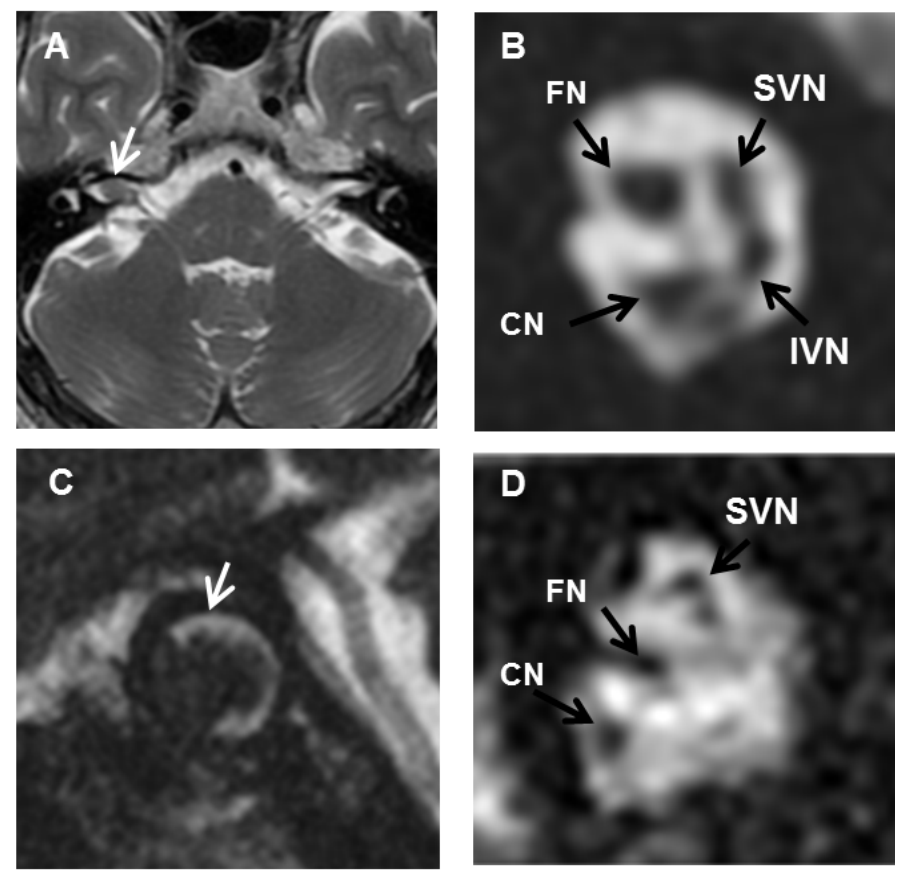

Figure 1. An intra-canalicular tumor in a 47-year-old patient with right sensorineural hearing loss (Patient 1).A) Axial T2-weighted MR image shows a filling defect within the high-signal CSF in the rightinternal auditory canal (IAC; white arrow). C) Oblique sagittal 3D-FIESTA MR image at the right porusacusticus clearly reveals a $5 \mathrm{~mm}$ intra-canalicular tumor (white arrow). (B,D) Oblique sagittal 3D-FIESTA MR images at the fundus of the IAC. Four nerves are clearly displayed in the image from the left side (B), while the nerves on the right side are only vaguely visible and the IVN is not observable (D).

CN: Cochlear Nerve; FN: Facial Nerve; IVN: Inferior Vestibular Nerve; SVN: Superior Vestibular Nerve. 


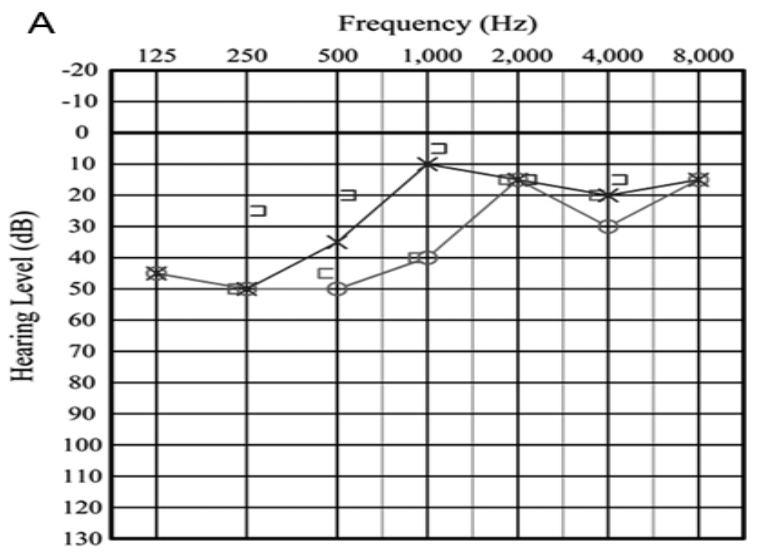

\section{B}

$(\%)$

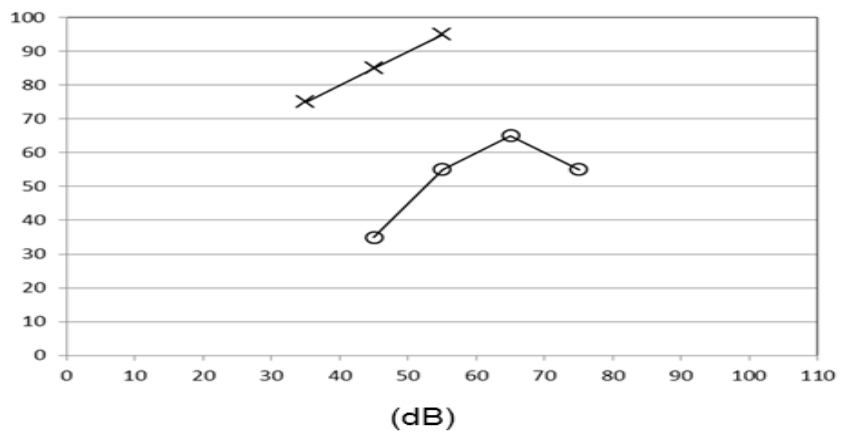

Figure 2. Audiological findings from a patient with a right-sided intra-canalicular tumor (Patient 1). A) Pure tone audiogram and, B) word recognition curve.
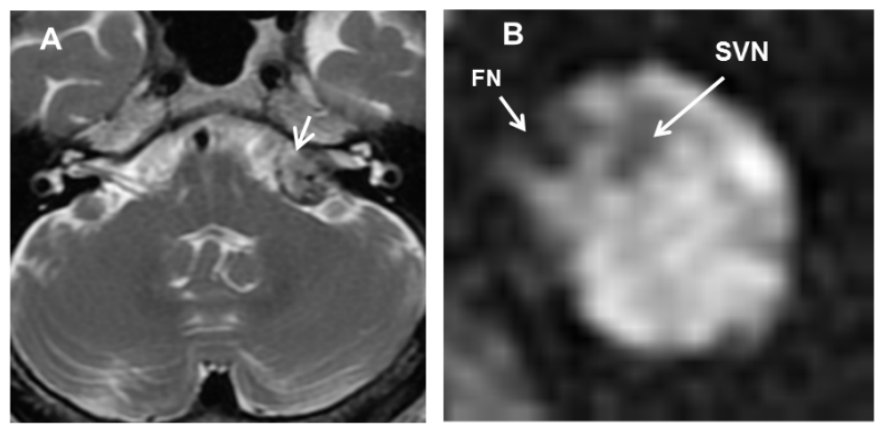

Figure 3. Cerebellopontine angle (CPA) tumor in a 59-year-old patient with left sensorineural hearing loss (Patient 2). A) Axial T2-weighted MR image shows a $15 \mathrm{~mm}$ CPA mass and an enlarged canal on the left side (white arrow). B) Oblique sagittal 3D-FIESTA MR image through the left internal auditory canal. The cochlear nerve and inferior vestibular nerve are highly atrophic and are undetectable. FN: Facial Nerve; SVN: Superior Vestibular Nerve.
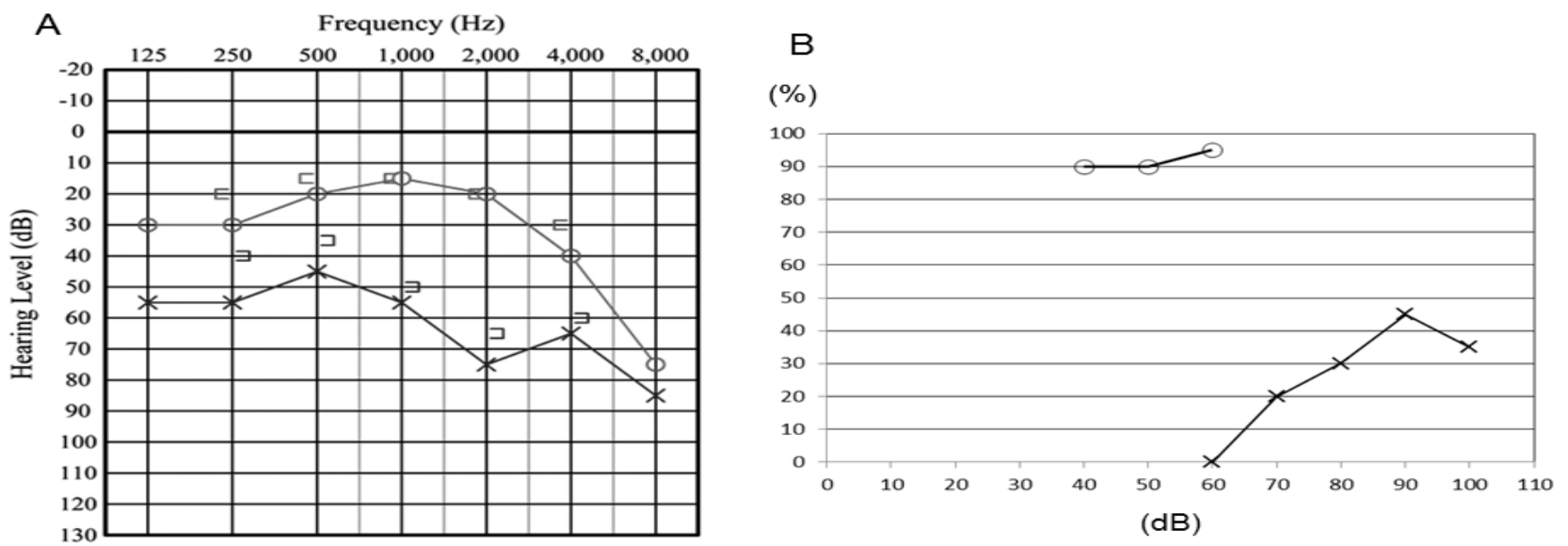

Figure 4. Audiological findings in a patient with a left-sided cerebellopontine angle tumor (Patient 2). A) Pure tone audiogram and, B) word recognition curve.

Table 1. Audiological, vestibular, and radiological findings in patients with acoustic schwannoma.

\begin{tabular}{|c|c|c|c|c|c|c|c|c|c|c|}
\hline \multirow[b]{2}{*}{ Case No. } & \multirow[b]{2}{*}{$\begin{array}{c}\text { Age (years) } \\
\text { /Sex }\end{array}$} & \multirow[b]{2}{*}{ Side } & \multicolumn{5}{|c|}{ Audiological findings in affected ear } & \multicolumn{2}{|c|}{ Vestibular Assessments } & \multirow{2}{*}{$\begin{array}{c}\text { MRI } \\
\begin{array}{c}\text { CN diameter } \\
(\mathbf{m m})\end{array}\end{array}$} \\
\hline & & & \begin{tabular}{|} 
Pure tone \\
hearing level \\
(dB)
\end{tabular} & $\begin{array}{l}\text { ABR thresholds } \\
\text { (dB nHL) }\end{array}$ & $\begin{array}{c}\text { ABR IT5 } \\
(\mathrm{ms})\end{array}$ & $\begin{array}{l}\text { Maximum speech } \\
\text { discrimination (\%) }\end{array}$ & DPOAE & Caroric test & ${ }_{0}$ VEMP & \\
\hline 1 & $47 / \mathrm{M}$ & $\mathrm{R}$ & 35 & 60 & 1.9 & 65 & present & DP & present & 0.8 \\
\hline 2 & $59 / \mathrm{M}$ & $\mathrm{L}$ & 62 & 70 & 1.2 & 45 & absent & $\mathrm{CP}$ & present & absent \\
\hline 3 & $62 / \mathrm{M}$ & $\mathrm{L}$ & 48 & - & - & 70 & absent & - & - & 0.6 \\
\hline
\end{tabular}

- : not evaluated; ABR: Auditory Brainstem Response; DPOAE: Distortion Product Optoacoustic Emission; F: Female; IT5: Interaural Differences in the Latencies of Wave V; M: Male; oVEMP: Ocular Vestibular-Evoked Myogenic Potential. 

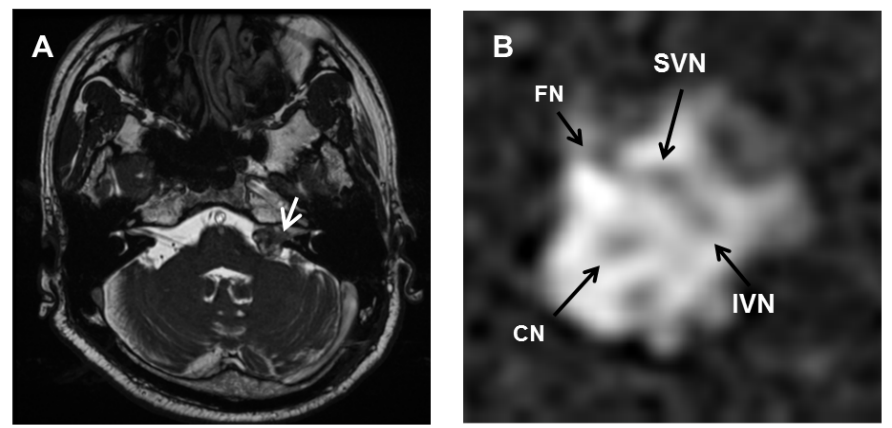

Figure 5. Cerebellopontine angle (CPA) tumor in a 59-year-old patient with left sensorineural hearing loss (Case 3). A) Axial T2-weighted MR image shows a 15 mm CPA mass and an enlarged canal on the left side (white arrow). B) Oblique sagittal 3D-FIESTA MR image through the left internal auditory canal. The left intra-canalicular nerves are atrophic.CN: Cochlear Nerve; FN: Facial Nerve; IVN: Inferior Vestibular Nerve; SVN: Superior Vestibular Nerve.

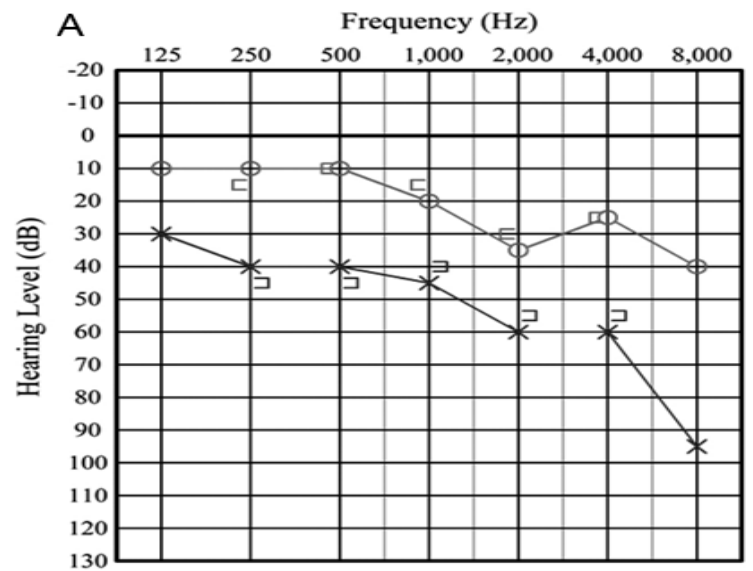

\section{B}

$(\%)$

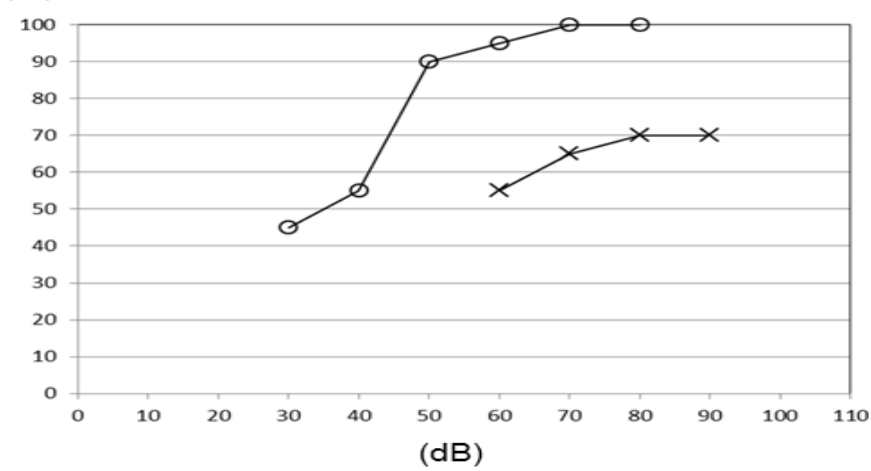

Figure 6. Audiological findings from a patient with a left-sided cerebellopontine angle tumor (Patient 3). A) Pure tone audiogram and, B) word recognition curve.

In contrast to the high $\mathrm{T} 2$ signal of cerebrospinal fluid, acoustic schwannomas show low T2 signal intensity [4]. Imaging of the temporal bone with 3D-FIESTA sequences in patients presenting with hearing loss, tinnitus, vertigo, and peripheral facial palsy has previously demonstrated positive findings in $18.37 \%$ of patients [5]. The CN, SVN, IVN, and FN all have a characteristic cross-sectional appearance on sagittal oblique FIESTA images [1], and 3D-FIESTA [6] and other SSFP sequences with high spatial resolution have been demonstrated as being significantly better for the identification of the four intracanalicular nerves than 3D turbo spin echo (TSE) sequences [7]. Due to its superior spatial resolution, the direct sagittal oblique imaging technique theoretically allows clearer delineation of the individual nerves of the IAC than can be achieved with MPR techniques [2]. Furthermore, MPR techniques are generally time-consuming in comparison with the direct imaging technique.

The $\mathrm{CN}$ is usually considered normal whenever its diameter is larger or equal to that of the FN [8], and several studies using SSFP MRI sequences have reported normal diameter values for the $\mathrm{CN}[3,8]$. In the study of Nakamichi et al. [3], the long and short diameters of the $\mathrm{CN}$ were approximately $1.35 \pm 0.16 \mathrm{~mm}$ and $0.99 \pm 0.18 \mathrm{~mm}$ when measured on the parasagittal image. In this report, the CN diameters in patients with acoustic schwannomas were small in comparison with those of previous reports $[3,8]$. Schwannomas rarely arise within the lateral IAC (fundus) [9], so in most subjects with acoustic schwannomas the $\mathrm{CN}$ diameter can be evaluated at the lateral IAC.

Acoustic schwannomas arise principally from the vestibular portion of the eighth cranial nerve. In over $90 \%$ of cases, these tumors arise from the IVN [9], as was revealed for Patient 1.
$\mathrm{CN}$ deficiency may be due to congenital hypoplasia of the nerve, acquired degeneration, or atrophy. Acquired CN deficiencies have been most commonly observed in patients with labyrinthitis ossificans. However, acquired CN deficiency with acoustic schwannoma has recently been reported [10]. Grastonbury et al. [10] designated CN deficiency as an undetectable $\mathrm{CN}$ on axial, coronal, or oblique sagittal MR images. However, a highly atrophic nerve below the limits of MRI resolution may appear to be absent. We therefore prefer the term hypoplasia rather than deficiency, and patients of $\mathrm{CN}$ hypoplasia with mild to moderate hearing loss have been reported previously [2]. In Patient 2, the $\mathrm{CN}$ in the affected side was not visible, but the averaged hearing level of the affected side was $58.3 \mathrm{~dB}$. An extremely atrophic and partially functional $\mathrm{CN}$ was therefore believed to be present in the affected side of this patient. This is the first report of acquired CN hypoplasia with moderate hearing loss to our knowledge.

Acoustic schwannomas typically present with progressive or suddenly deteriorating unilateral SNHL, which is presumably the result of vascular impairment or direct compression of $\mathrm{CN}$ fibers [10]. A previous study found that the audiometric pattern of the involved ears was high-frequency hearing loss in $65 \%$ of patients [11]. Poor speech discrimination, out of proportion to pure tone loss, may be an indicator of retrocochlear pathology. It has been reported that $45 \%$ of patients with acoustic schwannomas have abnormal speech discrimination abilities [11]. In Patient 1, the maximum word recognition score for the right ear was poor $(65 \%)$ when compared with that expected from pure tone hearing levels.

In ABR testing, the most reliable indicator for a diagnosis of acoustic schwannoma is an interaural difference in the latency of wave 
$\mathrm{V}$, known as IT5 [11]. Normative values of IT5 at $80 \mathrm{~dB} \mathrm{nHL}$ are $<0.4$ $\mathrm{ms}$. The prolonged IT5 found in Patients 1 and 2 , and the elevated wave $\mathrm{V}$ threshold in the affected side of Patient 1 , both indicate retrocochlear pathology. The prolonged IT5, poor speech discrimination, and preserved DPOAE responses of the affected ear in Patient 1 are suggestive of a retrocochlear disorder and a preservation of inner ear function. The SNHL of the affected ears in the other two cases was thought to be caused by combined inner ear and retrocochlear disorders.

Caloric testing assesses the function of the lateral semicircular canal, which is innervated by the SVN. Low caloric responses represent tumor involvement in the SVN [12]. The oVEMPs testing reflects the function of the utricle, which connects to the IVN. The absence of an oVEMP may possibly indicate tumor involvement of the IVN [4]. The 3D-FIESTA oblique sagittal MR images of the affected side in the patients of Patients 1 and 2 showed severe atrophy of the IVN in comparison with the FN and SVN. In these patients, the reduced or absent caloric responses with the presence of oVEMPs on the affected side are consistent with the IVN hypoplasia and preserved SVN observed in the 3D-FIESTA sequences. In Patient 1 , the IAC tumor was proven to be acoustic schwannoma originating from the IVN. In Patient 2, the IVN was also likely to be the site of origin. In Patient 3, the 3D-FIESTA sequences on the affected side showed that the SVN and IVN were almost equally atrophied, and the site of origin could not therefore be accurately estimated. It is generally accepted that hearing preservation is likely to be better in tumors originating from the SVN, rather than from the IVN [12].

In conclusion, oblique sagittal 3D-FIESTA MRI through the IAC can be obtained without MPR. The high spatial resolution direct imaging technique is useful for the identification of the four intracanalicular nerves, as it does not use reconstructions and provides shortening imaging time. In this work, we presented three cases of CPA/IAC tumors, and compared the MRI findings with the results of audiological and vestibular examinations. Acquired $\mathrm{CN}$ hypoplasia with moderate hearing loss was found in a patient of CPA tumor. In the patient of an acoustic schwannoma surgically demonstrated to have originated from the IVN, the results of the vestibular assessment indicated IVN pathology. The 3D-FIESTA MRI sequence may be helpful for planning surgery for acoustic schwannomas, but future improvements of the imaging technique for facial nerve preservation are desirable.

\section{Disclosure statement}

The authors report no conflicts of interest. The authors alone are responsible for the content and writing of the paper.

\section{Funding}

This work was not supported by any agency grant.

\section{References}

1. Sheth S, Branstetter BF 4th, Escott EJ (2009) Appearance of normal cranial nerves on steady-state free precession MR images. Radiographics 29: 1045-1055. [Crossref]

2. Taiji H, Morimoto N, Matsunaga T, (2012) Unilateral cochlear nerve hypoplasia in children with mild to moderate hearing loss. Acta Oto-Laryngologica 132: 1160-1167. [Crossref]

3. Nakamichi R, Yamazaki M, Ikeda M, Isoda H, Kawai H, et al. (2013) Establishing normal diameter range of the cochlear and facial nerves with 3D-CISS at 3T. Magn Reson Med Sci 12: 241-247. [Crossref]

4. Swartz JD (2008) Pathology of the vestibulocochlear nerves. Neuroimag Clin N Am 18: 321-346. [Crossref]

5. Cavusoglu M, Ciliz DS, Duran S, Ozsoy A, Elverici E, et al. (2016). Temporal bone MRI with 3D-FIESTA in the evaluation of facial and audiovestibular dysfunction Diagn Interc Imaging 87: 863-869. [Crossref]

6. Erdogan N, Altay C, Akay E, Karakas L, Uluc E, et al. (2013) MRI assessment of internal acoustic canal variations using 3D-FIESTA sequences. Eur Arch Otorhinolaryngol 270: 469-475. [Crossref]

7. Liang C, Zhang B, Wu L, Du Y, Wang X, et al. (2010). The superiority of 3D-CISS sequence in displaying the cistern segment of facial, vestibulocochlear nerves and their abnormal changes. Eur J Radiol 74: 437-440.

8. Jaryszak EM, Patel NA, Camp M, Mancuso AA, Antonelli PJ (2009) Cochlear neve diameter in normal hearing ears using high-resolution magnetic resonance imaging. Laryngoscope 119: 2042-2045. [Crossref]

9. Khrais T, Romano G, Sanna M (2008) Nerve origin of vestibular schwannoma: A prospective study. J Laryngol Otol 122: 128-132. [Crossref]

10. Glastonbury CM, Davidson HC, Harnsberger HR, Butker J, Kertesz TR, et al. (2002) Imaging findings of cochlear nerve deficiency. AJNR Am J Neuroradiol 23:635-643. [Crossref]

11. Stuken EZ, Brown K, Selesnick SH, (2012) Clinical and diagnostic evaluation of acoustic neuromas. Otolaryngol Clin N Am 45: 269-284. [Crossref]

12. Angeli S (2012) Middle fossa approach: Indications, Technique, and results. Otolaryngol Clin N Am 45: 417-438. [Crossref]

Copyright: (C2017 Taiji H. This is an open-access article distributed under the terms of the Creative Commons Attribution License, which permits unrestricted use, distribution, and reproduction in any medium, provided the original author and source are credited. 